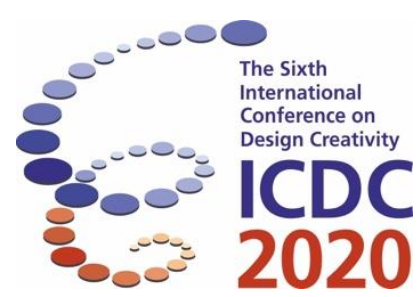

\title{
Empathy and Idea Generation: Exploring the Design of a Virtual Reality Controller for Rehabilitation Purposes
}

\author{
Yazan Barhoush $^{1}$, Georgi V. Georgiev ${ }^{1}$ and Brian Loudon ${ }^{2}$ \\ ${ }^{1}$ Center for Ubiquitous Computing, University of Oulu, Finland \\ ${ }^{2}$ Loud1Design Limited, Glasgow, UK
}

\begin{abstract}
The paper explores the ideation and design of a Virtual Reality (VR) proof-of-concept controller for rehabilitation of users with limited physical mobility (upper-limb disability). An existing tracker solution is used to map input (actions and movements) in VR. The main challenge was integrating some of the default functionalities existing in current commercial VR controllers, while providing an empathic setup and a use-case for disability rehabilitation, as well as keeping the controller compact, lightweight, and handheld. The prototyping process followed a human-centred explorative design idea generation. Only limited functionality of existing commercial controllers was maintained, with the feasibility and readiness for implementing additional functionalities to use the controller with existing applications and future use cases. An experiment was performed to investigate the usability of the system and the effectiveness and reliability of the controller in empathic remapping of real-life disability to VR.
\end{abstract}

Keywords: virtual reality, healthcare design, rehabilitation, empathetic design, human-centered design

\section{Introduction}

Research confirms that rehabilitation can improve cognitive functions (Kwakkel et al., 1999). The objective of rehabilitation is to 1) identify the means to provide repeated opportunities for tasks that further enable increases in motor system functions (the central nervous system is involved with movement) and 2) involve multimodal processes (different sensory modalities including vision, haptics, proprioception, audition) (Sveistrup, 2004). Effective rehabilitation aims at making improvements in people's daily self-care activities - this is also known as Activities of Daily Living (ADLs) - and depends on three factors, namely early intervention, task-oriented training, and repetition intensity (Malouin et al., 2003).

Virtual Reality's (VR) potential makes it an exciting safe tool for rehabilitation (Gustavo \& Mindy, 2011; Fluet \& Deutsch, 2013). Its applications are beginning to improve despite their newness (Crosbie et al., 2007). The quantity of low-cost multisensory VR applications has become increasingly accessible due to reductions in the costs, which can be attributed to Head Mounted Displays (HMDs), motion trackers as well as new haptic devices, encouraging the improvement of low-cost easy to use technologies (Sveistrup, 2004). Moreover, VR allows individuals to interact and train with or within interesting and relatively realistic three-dimensional (3D) environments (Sisto et al., 2002). These augmented Virtual Environments (VEs) were outlined as a therapy that promised ecologically valid 
intensive task-specific training (Rizzo et al., 2004). They provide multi-sensory training that would be transferred from the virtual world to the real world (Weiss et al., 2004).

Together, VR and VEs could deliver training intensity (repetitions and duration) associated with motor control, neural plasticity (the ability of the brain to change continuously throughout an individual's life (Duffau, 2016)), and positive behavioural adaptations (Adamovich et al., 2009; Sveistrup, 2004; Gustavo \& Mindy, 2011). The intensive repetition can offer effective rehabilitation while still being applicable to ADLs in a manner that can be more interesting and conducive to self-direction than conventional therapies (Nelles et al., 2001; Small \& Solodkin, 2016; Liepert Joachim et al., 2000; Broeren et al., 2002; Cramer \& Bastings, 2000).

Alternatively, although VR methods do support upper-limb rehabilitation, they still need to be further proved when compared to conventional therapies in use (Lucca, 2009; Levin et al., 2015). The value of VR rehabilitation needs further assessment (Crosbie et al., 2007). There exist unresolved questions in VR rehabilitation regarding technology (e.g. utilization of haptic interfaces to provide feedback), clinical application, selection of participants and training (e.g., cognitive and perceptual requirements for effective sensorimotor rehabilitation), and practical considerations (e.g. technology cost). To answer these questions, future studies are needed to tackle the issue of the effectiveness of virtual rehabilitation compared to standard care as well as to compare the effectiveness of the many approaches of presenting and delivering virtual interventions (Fluet \& Deutsch, 2013).

This paper contributes towards a design context of empathic design through idea generation, experimentation and development of custom controllers, as well as a physically optimized gaming VE to deliver an enhanced VR experiences for disability rehabilitation. The specific use case of this paper is upper-limb limited mobility rehabilitation. The controllers to be designed with this design context aim at providing a VR to real-life re-mapping of the disability for object interaction. The VR to Reallife re-mapping amplifies the limited range of motion of the user to allow for a larger spatial reach in VR space. The design of the controller, its interactive task, and the empathic setup design are explorative.

Current solutions in the market include Controller Switches, Accessibility Controllers and Interfaces for Gamers as well as Assistive Technologies that are specifically meant for VR rehabilitation. For example, OneSwitch provides ideas and solutions for switching users and supporting game accessibility solutions. They focus on accessibility switches - a simple on/off push button. Such switches can come in various shapes such as sound switches, movement switches, pressure mats, and pillow switches (OneSwitch.org.uk, n.d.). Another supplier solution provider, based in the UK, is Inclusive Technology. They provide software and hardware for people with special needs. Their assistive technology enables customers to communicate, learn, and play (e.g. switch access, mounting solutions, and environmental control) (Inclusive, n.d.). An example for an accessibility controller is the Xbox Adaptive Controller, which is a controller that targets gamers with limited mobility. The users can attach external devices such as switches, buttons, and joysticks to create a customizable controller's experience (Xbox, n.d.). When it comes interfaces for gamers, CyWee Z (CyWee Group Ltd) is a movement-based game controller used during intervention for users to play games. They reported improvements in performance in adults with upper-limb disability due to the repetitive, game-assisted, self-supported bilateral exercises (Hijmans et al., 2011). In another study, Fanucci et al. (2011) suggest a console interface targeting game accessibility for people with motor impairments. Their interface device allows people with upper-limb disabilities and people with mild cognitive disabilities to play with a popular game console, alone or with a mate, depending on the game and the user's capabilities (Fanucci et al., 2011).

There are also examples for Assistive Technologies for VR rehabilitation. WalkinVR Driver is a software program which aims to make VR applications accessible for everyone (e.g. users can move and rotate in VR without the need for moving in the real world) (WalkinVR, n.d.). SaeboVR focuses exclusively on improving patients' Activities of Daily Living (ADLs). It utilizes an on-screen virtual assistant that provided a real-time feedback (Saebo, 2017). The MindMotion PRO is solution for VE therapy for upper-limb neurorehabilitation (MindMotion, n.d.). 


\section{Design context, experimentation and development of controller}

The design process of the controller follows an explorative user-centred idea generation approach (Brown \& Katz, 2011; Newell \& Gregor, 2000). The approach was iterative in nature: rapid prototyping of different versions of the controller and quick user feedback on these controller prototypes were conducted. For example, the controller's casing (the electronics' housing) went through five iteration, where the housing was 3D printed and tested for 1) its capability to house the electronic components 2) its ergonomic design to make sure that the controller fits in hand. Additionally, the VR environment was designed 1) to be functional with the controller and 2) to include a the simple task that could have a rehabilitation potential (the grab activity).

The process applies and iterates general steps of the design thinking approach, empathize, define, ideate, prototype, and test (Brown \& Katz, 2011). The emphasis is on empathizing, prototyping, and testing with users. The user-centred design approach creates the fundamentals of further development of various controllers with the co-design approach involving persons with various disabilities, caregivers, rehabilitators, therapists, families, and other stakeholders having relevant roles in the well-being of persons with disabilities. Our design context of empathic setup involves: 1) generating ideas of possible re-mappings of real-life in VR for particular disability case; 2) empathic experimentation with these re-mappings, corresponding custom controllers and real-life interactions; 3) quantification of utility of solution for the particular disability case. Such development-based empathetic design will further respond to ergonomic design requirements (Rahman \& Sprigle, 1997) as well as emotional, cognitive, aesthetic, and social requirements in VR-based rehabilitation of each person's disability case (Howard, 2017).

A disability-simulating VR setup was needed to promote empathy (McDonagh et al., 2011) amongst healthy test users. Empathy is a relevant approach to broaden knowledge and understanding in the context of ensuring relevant outcomes of the design (McDonagh et al., 2011). Various design tools or design strategies focused on empathy help to identify authentic human needs (McDonagh \& Thomas, 2010). These are particularly applicable in cases of designing the related experiences and products. Moreover, empathic modelling (referring to experiencing with one's own body the physical situations of others (McDonagh \& Thomas, 2010)) is a design strategy that enables understanding and broadens a person's empathic horizon.

For this study, the idea of an empathic setup was inspired from looking into projects that are trying to offer a new perspective on what it is like to experience disability conditions or aging. For example, an MIT VR project was designed to simulate scuba diving (Couch, n.d.). To simulate the experience, the users wear a VR HMD and special gloves while lying on a device where their arms and legs are held aloft by elastic straps. Another example system, AGNES (Age Gain Now Empathy System), is a suit that can be used to understand better the physical challenges associated with ageing (AGNES $\mid$ MIT $A g e L a b$, n.d.). It has been adjusted to approximate the motor, visual, flexibility, dexterity, and strength of an elderly person (e.g. elastic straps are used to limit arm movement). Adhering to these two example systems, a use case was built to realize a simulated empathetic disability experience to mimic an upperlimb disability condition; arm movement was limited while using the fabricated controller by introducing chains of springs. The users need to apply force to open their arm (force is applied to expand the springs).

Additionally, to re-map and track movements of the controller in VR, the HTC VIVE Tracker was used. Existing systems include the HTC VIVE Tracker implemented in their VR controllers' designs, but without targeting disability rehabilitation. Choi et al. (2018) prototyped a handheld VR controller with a typical controller functionality, force feedback and actuated movement to the index finger. The controller allowed for three distinct interactions: 1) grasping a virtual object, 2) touching a virtual surface and 3) triggering (Newell \& Gregor, 2000). Murray et al. (2018) presented an entirely compliant controller handle for use in VR. The controller handles transitions between two static states: 1) a semirigid, large diameter state when pressurized and 2) a soft, compressible, smaller diameter state when depressurized. The two controller states are used to simulate the physical feel of two virtual objects (Murray et al., 2018). Another handheld VR controller that renders fingertip haptics when interacting with virtual surfaces is presented by Whitmire and colleagues (2018), where they evaluated the controller in two studies to see how the wheel speed and direction could impact the perceived realism 
in three application scenarios. An open source Etextile data glove was designed to facilitate detailed manipulation of 3D objects in VR, was described by Glowacki et al., (2019). The gloves detect when a user pinches his/her thumb and index finger, or thumb, and middle finger.

\section{Experimental setup}

An experiment was performed to investigate: 1) The usability and UX of the system 2) The effectiveness and reliability of the controller and the setup in empathic re-mapping of different VR real-life levels for disability rehabilitation.

To investigate these two objectives, 12 participants were recruited (six women and six men) with ages ranging from 20 to 30 years $(\mathrm{SD}=2.53$ ). All the recruits were healthy users and right-handed. All the participants were students at the University of Oulu from various fields and faculties. The participants seated on a chair facing a table. They wore the elastic band and springs were attached. The controller and the physically simulated VE, shown in Figure 1, were presented to them.

The scope of the project was explained to them; they understood the purpose of the controller, the idea of re-mapping, and the empathetically introduced disability simulation. Two different spatial scaling factors were used in two different sessions in order to amplify the limited range of motion of the user, to allow for larger spatial reaches in VR space. The VE interaction consisted of a set of balls on a big table, which can be grabbed by the user using the controller and placed on smaller tables. Since repetition is crucial for rehabilitation, the participants were required to repeat the task for three times. Each participant would perform the experiment for 15 minutes, fill out questionnaires, and answer interview questions. A coffee voucher was given as a gift for participation. The study lasted approximately 30 minutes.

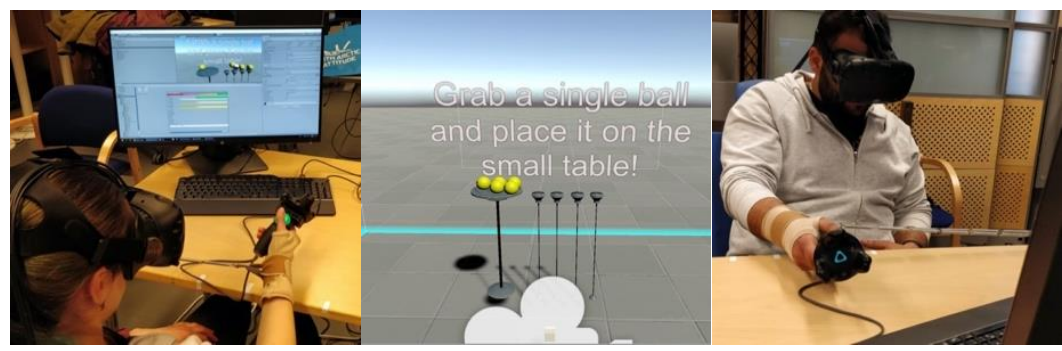

Figure 1. Participant's seating, the physically simulated VE for the Task, Session in the VE; a cable, instead of battery, was used in the experiment so that the battery does not deplete

\section{Results and discussion}

The questionnaires were used for measuring the usability of the controller, evaluating the UX in a VR setting and getting an insight into the effectiveness and reliability of the controller in empathic remapping of different VR-real-life levels for disability rehabilitation.

\subsection{User experience and usability}

AttrakDiff helps to understand how the users personally rate the usability and design of an interactive product. In the AttrakDiff questionnaire, both hedonic and pragmatic dimensions of UX are studied with semantic differentials. A single evaluation scenario where the product was evaluated by the users only once (AttrakDiff, n.d.). Figure 2 shows the three results diagrams.

The Portfolio of results shows that the prototype was rated well in both Hedonic Quality (HQ) and Pragmatic Quality (PQ) - it was regarded as "desired." The results show that the PQ value is greater than HQ (PQ: 1.56, Confidence: 0.97; HQ: 1.29, Confidence: 0.57), which makes the product pragmatic. However, there is still room for optimization; the confidence rectangle extends from the desired area and into self-oriented, neutral, and task-oriented areas. Therefore, it cannot clearly be classified as desirable. In terms of PQ and HQ, the character classification does not apply because the confidence interval spills out over the character zone. The product assists its users optimally. However, $\mathrm{PQ}$ is only average. There exists a room for improvement in terms of PQ. Alternatively, the user is 
simulated by this product. However, the HQ value is only average. There exists a room for improvement in terms of HQ. The large confidence interval for both HQ and PQ could be attributed to the age range that is quite small $(\mathrm{SD}=2.5 \mathrm{y})$ in the considered range of participants (20-30). We believe that this variety depends on the small sample (12 people) and the contrasting "backgrounds": like different majors or faculties.
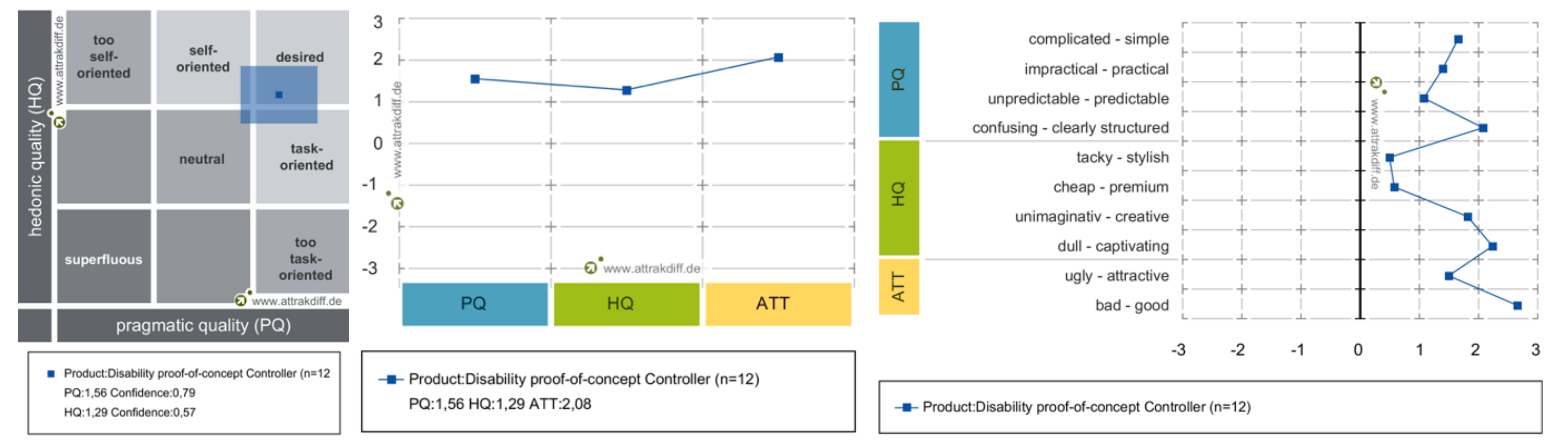

Figure 2. A) Portfolio of results: HQ is on the vertical axis, and PQ is on the horizontal. The product is placed in one or more "character-regions" depending on the values. The confidence is represented with a rectangle; B) Diagram of average values; C) Diagram of average values: HQ distinguishes between the aspects of stimulation and identity. The rating of Attractiveness (ATT) is presented.

When it comes to the Diagram of average values, for both PQ and HQ, the product is located in the above-average region. It meets more than just ordinary standards. However, an improvement is required to bind the users more to the product. Additionally, to motivate and simulate the users more intensely, further improvement must be aimed. The product's ATT value is in the above-average region-this gives the overall impression that the product is very attractive.

The Description of word-pairs shows the extreme values are of particular interest; they represent which characteristics are particularly critical or well-resolved. All mean scores reside on the right side (positive region) of the vertical scale, describing the product as "clearly structured," "captivating," and "good" in terms of PQ, HQ, and ATT, respectively.

\subsection{Presence}

The environment experience in VR was validated by measuring the degree of immersion and realism offered by the test scene. The Witmer \& Singer presence questionnaire (PS) (Witmer et al., 2005; Witmer \& Singer, 1998) is a widely used technique to measure such experiences in VEs. They define presence as "the subjective experience of being in one place or environment, even when one is physically situated in another".

The PS considers several factors that are thought to influence presence. The main factors are control, sensory distraction, and realism. The questionnaire is often customized to fit the requirements of the study (e.g., https://edutechwiki.unige.ch/en/Presence/Presence_Questionnaire_(PQ)). It consisted of 19 items to be answered on a seven-point Likert scale. The questionnaire was revised to adapt to our VE. Certain items of the original PS were dropped since they are unrepeatable to our system or the experimental setup (e.g. auditory fidelity). Two additional questions that were added: 1) How well were you able to pick up an object in the Virtual Environment? 2) How well was the introduced disability simulated?

According to the typical scoring of PS, the total and average values are reported for five aggregated categories: realism, the ability to act, the quality of the interface, the ability to examine, and selfevaluation of performance. The Values are plotted in Figure 3. All average scores were all within the positive range (4-7). Examining the participants' comments from the evaluation, it is noted that the interacting with objects was a favourable task. The Participants gave positive comments, such as "Nice work. If I ever break a hand, I will use this piece of software to get back on track!" and "Nice experience." A few other recommendations to improve the system were also mentioned: "The balls kind of looked blurry probably due to the headset" and "The button is quite sensitive and it could be activated 
without putting any pressure. Also, it is a bit unbalanced. The weight in the front bends my wrist involuntary. Maybe a counterweight on the back would balance it out".

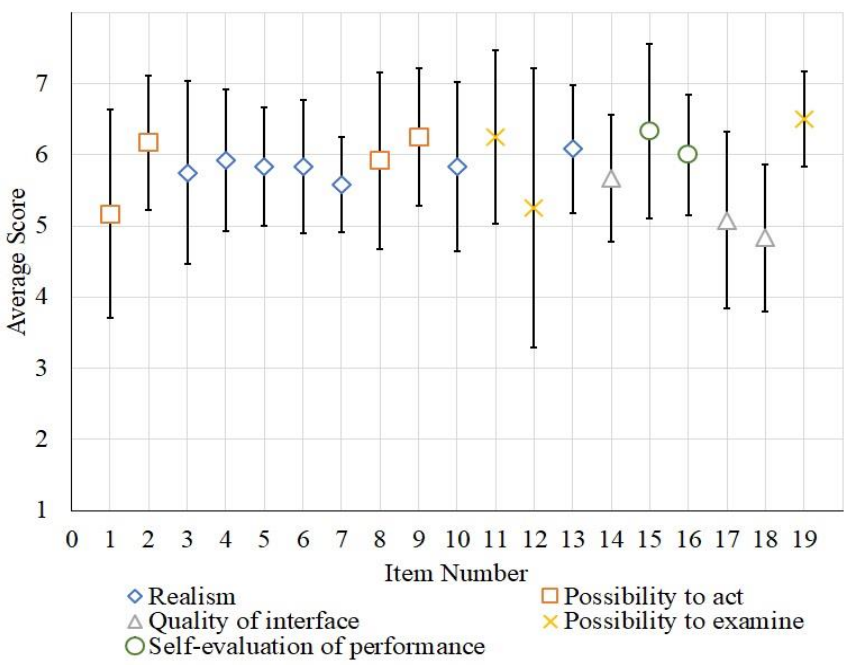

Figure 3. PS results, the 19 items are the questions, standard deviation is used to represent error

\section{Summary and insights}

This paper contributes toward the development of a design context through idea generation, empathic experimentation and development of custom VR controllers, to deliver enhanced VR experiences for rehabilitation purposes. The application case of a proof-of-concept controller provided a VR to real-life re-mapping for object interaction. This design context is promising since it provides flexibility in customization and may provide a means for a wide spectrum of creative implementations in the future.

\subsection{Limitations}

Even though the controller design went through several iterations, it still has several limitations. Overall the controller and its current housing materials give a cheap and unfinished impression due to the prototyping nature of their construction. The shape was generated for speed of prototyping (this application case aimed at an empathetic explorative design for future studies). The models for the docking mechanism, however, are reusable for future applications and controllers that would involve the same tracker.

When it comes to the limitations on the user's movements when simulating the disability, springs were chosen to make easier re-mapping for the empathetic setup. There are no measures for how closely this simulates the experience of a given known disability, nor it was possible to verify the simulation against the experience of someone living with upper-limb issues. Since springs were attached to the forearm, the user could still utilize their wrists to help overcome the introduced disability. Although the user's movements were meant to be along the $\mathrm{x}$-axis only, it was not necessarily the case during the experiment: The users could slightly move along all axes when moving the grabbed ball from one table to another. Only one axis remapping was desired to keep the experiment simple. Future experiments should having more complex re-mappings along all the axes.

\subsection{Future implications}

In future works, we aim to go from a generic design process, which introduces elements to facilitate the empathic involvement of participants, to deal farther with creativity. Additionally, the explored design context for empathic design is aimed to be more suitable for various conditions and adaptable to different specific situations in the future.

The developed controller can be used with existing applications. However, the future use cases it is recommended to create a custom rehabilitation environment. Moreover, developing a control interface for such controllers and corresponding interaction paradigms is aimed for in the future. Finally, it is 
intended to analyse and improve the hardware and software performance of the controller and corresponding environment.

\section{Acknowledgement}

This project has received funding from the European Union's Horizon 2020 research and innovation programme under grant agreement No 856998. This research has been also partially financially supported by Academy of Finland 6Genesis Flagship (grant 318927).

\section{References}

Adamovich, S. V., Fluet, G. G., Tunik, E., \& Merians, A. S. (2009). Sensorimotor training in virtual reality: A review. NeuroRehabilitation, 25(1), 29-44. https://doi.org/10.3233/NRE-2009-0497

AGNES (Age Gain Now Empathy System) | MIT AgeLab. (n.d.). Retrieved January 11, 2020, from https://agelab.mit.edu/agnes-age-gain-now-empathy-system

AttrakDiff. (n.d.). Retrieved January 11, 2020, from http://attrakdiff.de/index-en.html

Broeren, J., Björkdahl, A., Pascher, R., \& Rydmark, M. (2002). Virtual Reality and Haptics as an Assessment Device in the Postacute Phase after Stroke. CyberPsychology \& Behavior, 5(3), 207-211. https://doi.org/10.1089/109493102760147196

Brown, T., \& Katz, B. (2011). Change by Design. Journal of Product Innovation Management, 28(3), 381-383. https://doi.org/10.1111/j.1540-5885.2011.00806.x

Choi, I., Ofek, E., Benko, H., Sinclair, M., \& Holz, C. (2018). CLAW: A Multifunctional Handheld Haptic Controller for Grasping, Touching, and Triggering in Virtual Reality. Proceedings of the 2018 CHI Conference on Human Factors in Computing Systems, 654:1-654:13. https://doi.org/10.1145/3173574.3174228

Couch, C. (n.d.). A VR scuba diving project shows you an undersea world while helping you feel what it's like to be deaf. MIT Technology Review. Retrieved January 11, 2020, from https://www.technologyreview.com/s/601336/disability-simulating-vr-promotes-empathy/

Cramer, S. C., \& Bastings, E. P. (2000). Mapping clinically relevant plasticity after stroke. Neuropharmacology, 39(5), 842-851. https://doi.org/10.1016/S0028-3908(99)00258-0

Crosbie, J. H., Lennon, S., Basford, J. R., \& McDonough, P. S. M. (2007). Virtual reality in stroke rehabilitation: Still more virtual than real. Disability and Rehabilitation, 29(14), 1139-1146. https://doi.org/10.1080/09638280600960909

Duffau, H. (2016). Chapter 18-Brain Plasticity and Reorganization Before, During, and After Glioma Resection. In Glioblastoma (pp. 225-236). Elsevier. https://doi.org/10.1016/B978-0-323-47660-7.00018-5

Fanucci, L., Iacopetti, F., \& Roncella, R. (2011). A console interface for game accessibility to people with motor impairments. 2011 IEEE International Conference on Consumer Electronics -Berlin (ICCE-Berlin), 206-210. https://doi.org/10.1109/ICCE-Berlin.2011.6031883

Fluet, G. G., \& Deutsch, J. E. (2013). Virtual Reality for Sensorimotor Rehabilitation Post-Stroke: The Promise and Current State of the Field. Current Physical Medicine and Rehabilitation Reports, 1(1), 9-20. https://doi.org/10.1007/s40141-013-0005-2

Glowacki, B. R., Freire, R., Thomas, L. M., O’Connor, M., Jamieson-Binnie, A., \& Glowacki, D. R. (2019). An open source Etextile VR glove for real-time manipulation of molecular simulations. ArXiv:1901.03532 [Physics]. http://arxiv.org/abs/1901.03532

Gustavo, S., \& Mindy, L. (2011). Virtual Reality in Stroke Rehabilitation. Stroke, 42(5), 1380-1386. https://doi.org/10.1161/STROKEAHA.110.605451

Hijmans, J. M., Hale, L. A., Satherley, J. A., McMillan, N. J., \& King, M. J. (2011). Bilateral upper-limb rehabilitation after stroke using a movement-based game controller. The Journal of Rehabilitation Research and Development, 48(8), 1005. https://doi.org/10.1682/JRRD.2010.06.0109

Howard, M. C. (2017). A meta-analysis and systematic literature review of virtual reality rehabilitation programs. Computers in Human Behavior, 70, 317-327. https://doi.org/10.1016/j.chb.2017.01.013

Inclusive. (n.d.). Inclusive Technology-All the Help You Need. Retrieved October 22, 2019, from https://www.inclusive.co.uk/

Kwakkel, G., Wagenaar, R. C., Twisk, J. W., Lankhorst, G. J., \& Koetsier, J. C. (1999). Intensity of leg and arm training after primary middle-cerebral-artery stroke: A randomised trial. The Lancet, 354(9174), 191-196. https://doi.org/10.1016/S0140-6736(98)09477-X

Levin, M. F., Weiss, P. L., \& Keshner, E. A. (2015). Emergence of Virtual Reality as a Tool for Upper Limb Rehabilitation: Incorporation of Motor Control and Motor Learning Principles. Physical Therapy, 95(3), 415-425. https://doi.org/10.2522/ptj.20130579 
Liepert Joachim, Bauder Heike, Miltner Wolfgang H. R., Taub Edward, \& Weiller Cornelius. (2000). TreatmentInduced Cortical Reorganization After Stroke in Humans. Stroke, 31(6), 1210-1216. https://doi.org/10.1161/01.STR.31.6.1210

Lucca, L. F. (2009). Virtual reality and motor rehabilitation of the upper limb after stroke: A generation of progress? Journal of Rehabilitation Medicine, 41(12), 1003-1100. https://doi.org/10.2340/16501977-0405

Malouin, F., Richards, C. L., McFadyen, B., \& Doyon, J. (2003). Nouvelles perspectives en réadaptation motrice après un accident vasculaire cérébral. médecine/sciences, 19(10), 994-998. https://doi.org/10.1051/medsci/20031910994

McDonagh, D., \& Thomas, J. (2010). Disability + Relevant Design: Empathic Design Strategies Supporting More Effective New Product Design Outcomes. The Design Journal, 13(2), 180-198. https://doi.org/10.2752/175470710X12735884220899

McDonagh, D., Thomas, J., Khuri, L., Sears, S. H., \& Peña-Mora, F. (2011). Empathic Design Research Strategies: Designing For, With and By People with Disabilities. In Handbook of Research on Trends in Product Design and Development: Technological and Organizational Perspectives (pp. 58-79). IGI Global. https://doi.org/10.4018/978-1-61520-617-9.ch004

MindMotion. (n.d.). 3D Virtual Environment solutions for early motor rehabilitation. MindMaze. Retrieved October 22, 2019, from https://www.mindmaze.com/mindmotion/

Murray, B. C. M., Peele, B. N., Xu, P., Spjut, J., Shapira, O., Luebke, D., \& Shepherd, R. F. (2018). A variable shape and variable stiffness controller for haptic virtual interactions. 2018 IEEE International Conference on Soft Robotics (RoboSoft), 264-269. https://doi.org/10.1109/ROBOSOFT.2018.8404930

Nelles, G., Jentzen, W., Jueptner, M., Müller, S., \& Diener, H. C. (2001). Arm Training Induced Brain Plasticity in Stroke Studied with Serial Positron Emission Tomography. NeuroImage, 13(6), 1146-1154. https://doi.org/10.1006/nimg.2001.0757

Newell, A. F., \& Gregor, P. (2000). "User Sensitive Inclusive Design"-In Search of a New Paradigm. Proceedings on the 2000 Conference on Universal Usability, 39-44. https://doi.org/10.1145/355460.355470

OneSwitch.org.uk. (n.d.). About OneSwitch.org.uk. OneSwitch.Org.Uk. Retrieved October 22, 2019, from http://oneswitch.org.uk/page/about

Rahman, M. M., \& Sprigle, S. (1997). Physical Accessibility Guidelines of Consumer Product Controls. Assistive Technology, 9(1), 3-14. https://doi.org/10.1080/10400435.1997.10132291

Rizzo, A. A., Schultheis, M., Kerns, K. A., \& Mateer, C. (2004). Analysis of assets for virtual reality applications in neuropsychology. Neuropsychological Rehabilitation, $207-239$. https://doi.org/10.1080/09602010343000183

Saebo. (2017, July 11). Benefits of Virtual Reality for Stroke Rehabilitation. Saebo. https://www.saebo.com/benefits-virtual-reality-stroke-rehabilitation/

Sisto, S. A., Forrest, G. F., \& Glendinning, D. (2002). Virtual Reality Applications for Motor Rehabilitation After Stroke. Topics in Stroke Rehabilitation, 8(4), 11-23. https://doi.org/10.1310/YABD-14KA-159P-MN6F

Small, S. L., \& Solodkin, A. (2016). Review: The Neurobiology of Stroke Rehabilitation: The Neuroscientist. https://doi.org/10.1177/107385849800400614

Sveistrup, H. (2004). Motor rehabilitation using virtual reality. Journal of NeuroEngineering and Rehabilitation, 1(1), 10. https://doi.org/10.1186/1743-0003-1-10

WalkinVR. (n.d.). WalkinVR Driver-Accessibility for Virtual Reality. Virtual Reality with Disability, on Wheelchair, in Bed or without Hand - WalkinVR. Retrieved October 22, 2019, from https://www.walkinvrdriver.com/

Weiss, P. L., Rand, D., Katz, N., \& Kizony, R. (2004). Video capture virtual reality as a flexible and effective rehabilitation tool. Journal of NeuroEngineering and Rehabilitation, 1(1), 12. https://doi.org/10.1186/1743-0003$1-12$

Whitmire, E., Benko, H., Holz, C., Ofek, E., \& Sinclair, M. (2018). Haptic Revolver: Touch, Shear, Texture, and Shape Rendering on a Reconfigurable Virtual Reality Controller. Proceedings of the 2018 CHI Conference on Human Factors in Computing Systems, 86:1-86:12. https://doi.org/10.1145/3173574.3173660

Witmer, B. G., Jerome, C. J., \& Singer, M. J. (2005). The Factor Structure of the Presence Questionnaire. Presence: Teleoperators and Virtual Environments, $298-312$. https://doi.org/10.1162/105474605323384654

Witmer, B. G., \& Singer, M. J. (1998). Measuring Presence in Virtual Environments: A Presence Questionnaire. Presence: Teleoperators and Virtual Environments, 7(3), 225-240. https://doi.org/10.1162/105474698565686 Xbox. (n.d.). Xbox Adaptive Controller | Xbox. Xbox.com. Retrieved October 22, 2019, from https://www.xbox.com/fi-FI/xbox-one/accessories/controllers/xbox-adaptive-controller 